\title{
Fate of Midbrain Dopaminergic Neurons Controlled by the Engrailed Genes
}

\author{
Horst H. Simon, ${ }^{1}$ Harald Saueressig, ${ }^{1}$ Wolfgang Wurst, ${ }^{2,3}$ Martyn D. Goulding, ${ }^{1}$ and Dennis D. M. O'Leary ${ }^{1}$ \\ ${ }^{1}$ Molecular Neurobiology Laboratory, The Salk Institute, La Jolla, California 92037, ${ }^{2}$ Max Planck Institute for \\ Psychiatry, D-80804 Munich, Germany, and ${ }^{3}$ GSF-Research Center, Institute for Mammalian Genetics, D-85758 \\ Oberschleißheim, Germany
}

Deficiencies in neurotransmitter-specific cell groups in the midbrain result in prominent neural disorders, including Parkinson's disease, which is caused by the loss of dopaminergic neurons of the substantia nigra. We have investigated in mice the role of the engrailed homeodomain transcription factors, En-1 and En-2, in controlling the developmental fate of midbrain dopaminergic neurons. En-1 is highly expressed by essentially all dopaminergic neurons in the substantia nigra and ventral tegmentum, whereas En-2 is highly expressed by a subset of them. These neurons are generated and differentiate their dopaminergic phenotype in En-1/En-2 double null mutants, but disappear soon thereafter. Use of an En-1/tau-LacZ knock-in mouse as an autonomous marker for these neurons indicates that they are lost, rather than that they change their neurotransmitter phenotype. A single allele of En-1 on an En-2 null background is sufficient to produce a wild type-like substantia nigra and ventral tegmentum, whereas in contrast a single allele of
En-2 on an En-1 null background results in the survival of only a small proportion of these dopaminergic neurons, a finding that relates to the differential expression of En-1 and En-2. Additional findings indicate that En-1 and En-2 regulate expression of $\alpha$-synuclein, a gene that is genetically linked to Parkinson's disease. These findings show that the engrailed genes are expressed by midbrain dopaminergic neurons from their generation to adulthood but are not required for their specification. However, the engrailed genes control the survival of midbrain dopaminergic neurons in a gene dose-dependent manner. Our findings also suggest a link between engrailed and Parkinson's disease.

Key words: $\alpha$-synuclein; En-1; En-2; neuronal death; neuronal specification; dopamine; Parkinson's disease; substantia nigra; mouse; transcription factors; tyrosine hydroxylase; ventral tegmentum; tau-LacZ
The enormous variety of neuronal types in the nervous system becomes specified during development in a stepwise process (Tanabe and Jessell, 1996) from an originally uniform pool of neuroepithelial cells (Sechrist and Bronner-Fraser, 1991). Overlapping signals lay down the rostrocaudal and dorsoventral neuraxes of the embryo in a chronological order and determine where specific neuronal types will form (Simon et al., 1995). During this process, unique sets of transcription factors are activated in subsets of neuroepithelial cells and their progeny, and they specify neuronal phenotype. For example, the distinct subsets of spinal motor neurons and their progenitors are determined by their expression of unique combinations of Lim homeodomain transcription factors (Pfaff et al., 1996).

Engrailed, a homeodomain transcription factor, has well defined roles in insect development. During early development, engrailed establishes the body plan, defining for example the posterior half of each parasegment (Kornberg, 1981a,b; Poole

\footnotetext{
Received Dec. 8, 2000; revised Feb. 12, 2001; accepted Feb. 23, 2001.

This work was supported by National Institutes of Health Grants R01 NS31558 (D.D.M.O.) and R01 NS37075 (M.D.G.), Eu Biotech Grant PL960146 (W.W.), Human Frontier Science Program (HFSP) Grant RG-83/96 (W.W.), and fellowships NIH F32 NS10284 (H.H.S.), HFSP LT-526/94 (H.H.S.), and Deutsche Forschungsgemeinschaft SA652/2-1 (H.S.). We are grateful to A. Joyner for providing breeding pairs of En-1 and En-2 deficient mice, En-1 and En-2 cDNA, and the $\alpha$ Enhb antibody. We thank J.-P. Pinaud for technical assistance and R. Dyck and V. Blanquet for helpful discussions.

Correspondence should be addressed to Dennis D. M. O'Leary, Molecular Neurobiology Laboratory, The Salk Institute, 10010 N. Torrey Pines Road, La Jolla, CA 92037. E-mail: doleary@salk.edu.

Copyright (C) 2001 Society for Neuroscience 0270-6474/01/213126-09\$15.00/0
}

and Kornberg, 1988). Later, engrailed determines neuronal identity. For example, engrailed controls the glial-neuronal fate decision of certain multipotent neuroblasts in the grasshopper CNS (Condron et al., 1994) and of midline serotonergic neurons in Drosophila (Lundell et al., 1996).

Vertebrates have two engrailed homologs, En-1 and En-2, that are expressed during development in a domain encompassing the posterior midbrain and anterior hindbrain (Davidson et al., 1988; Gardner et al., 1988). This domain coincides with parts of the neural tube that generate dorsal structures, including the cerebellum and colliculi, as well as ventral midbrain nuclei. Targeted deletion of $E n-1$ and $E n-2$ in mice reveals their role in regulating the development of dorsal structures originating within their expression domain. En-1 mutants, which die on the day of birth, lack the cerebellum and inferior colliculus (Wurst et al., 1994). En-2 mutants are viable and fertile and have minor defects in cerebellar foliation (Joyner et al., 1991; Millen et al., 1994). Overexpression of En-1 and En-2 in chick midbrain indicates that engrailed regulates the anterior-posterior polarity of the optic tectum, the homolog of the mammalian superior colliculus (Friedman and O'Leary, 1996; Itasaki and Nakamura, 1996; Logan et al., 1996). Although these studies show that engrailed has a prominent role in vertebrate neural development, a function for engrailed in vertebrate neuronal specification has only recently been suggested (Saueressig et al., 1999).

The ventral midbrain nuclei, substantia nigra (SN) and ventral tegmentum (VT), are the most prominent sources of dopaminergic neurons in the CNS (German and Manaye, 1993). Degener- 
ation of these neurons and their projections to the basal ganglia and frontal cortex is implicated in several CNS disorders, including Parkinson's disease (PD), which is characterized by a debilitating loss of motor control (Polymeropoulos et al., 1997). The expression domains of En-1 and En-2 approximate the location of these neurons (Davis and Joyner, 1988; Davis et al., 1988), suggesting that engrailed may regulate their specification and differentiation. To investigate this issue, we related the expression of En-1 and En-2 to dopaminergic neurons and addressed the requirement of engrailed for their developmental fate in En-1 and En-2 mutant mice using cell autonomous and neurotransmitterspecific markers. Our findings led us to show that expression of $\alpha$-synuclein, a gene genetically linked to PD (Polymeropoulos et al., 1997; Kruger et al., 1998), is dependent on engrailed.

\section{MATERIALS AND METHODS}

Animals. Generation of the En-1- and En-2-deficient mice by targeted gene deletion has been described previously (Joyner et al., 1991; Wurst et al., 1994). The En-1/tau-LacZ mice were generated by a "knock-in" strategy in which the first 71 codons, including the start codon, were replaced by a tau-LacZ sequence (Callahan and Thomas, 1994) and resulted in an En-1 null allele. The construct and procedures are described in detail elsewhere (Saueressig et al., 1999). Parental lines for producing the mutant mice deficient for both $E n-1$ and $E n-2$ were kept as En $-1^{+/-} / E n-2^{-1-}$ or En-1/tau-LacZ $Z^{+/-} / E n-2^{-/-}$. A minimum of four mice of a given genotype, but usually more, were analyzed for each finding described. Each set of findings described for a given genotype and methodology was observed in each animal analyzed.

Immunohistochemistry on sections. The animals were perfused with $4 \%$ paraformaldehyde in $100 \mathrm{~mm}$ phosphate buffer (PB), pH 7.4, and immersion-fixed overnight at $4^{\circ} \mathrm{C}$. The brains were cryoprotected with $30 \%$ sucrose in $100 \mathrm{~mm}$ PB and cut at $50 \mu \mathrm{m}$ with a sledge microtome. Alternatively, the brains were embedded in an albumin-gelatin mixture as follows: $3 \%$ gelatin bloom 100 was heated until dissolved and cooled to $<40^{\circ} \mathrm{C}$, chicken egg albumin was added to a concentration of $30 \%$, and then the mixture was filtered through a $100 \mu \mathrm{m}$ nylon mesh. The tissue was put into a plastic mold containing the albumin-gelatin mixture and exposed to formaldehyde vapor overnight at $4{ }^{\circ} \mathrm{C}$ (formalin in a glass tray). When the top was firm, a $4 \%$ paraformaldehyde solution was poured into the mold and left overnight at $4^{\circ} \mathrm{C}$. The next day, the block was trimmed and immersed overnight in $4 \%$ paraformaldehyde. Then the specimen was sectioned with a vibratome at $70 \mu \mathrm{m}$.

The sections were immunostained in a plastic chamber; blocked with $10 \%$ heat-inactivated newborn calf serum (NCS), $1 \% \mathrm{H}_{2} \mathrm{O}_{2}, 1 \%$ Triton $\mathrm{X}-100$ in PBS for $1 \mathrm{hr}$ at room temperature; washed with PBT (PBS, $1 \%$ Triton X-100) three times for 10 min each; and incubated with primary antibody in PBT, 10\% NCS [rabbit anti-tyrosine hydroxylase (TH) 1:2000 (Chemicon, Temecula, CA); rabbit anti- $\beta$-galactosidase $(\beta$-gal) 1:10,000 (Organon Tecknika-Cappel, Durham, NC); or goat anti- $\beta$ galactosidase 1:10,000 (Arnel Products Co., New York, NY)] at $4^{\circ} \mathrm{C}$ overnight. They were washed three times with PBT for $10 \mathrm{~min}$, incubated with secondary antibody at 1:500 in PBT, 10\% NCS [biotinylated goat anti-rabbit, all secondary antibodies from Jackson ImmunoResearch (West Grove, PA)] for $2 \mathrm{hr}$, washed three times with PBT, and incubated with streptavidin-peroxidase (Jackson ImmunoResearch) at 1:1000. The sections were washed three times with PBT and twice with PBS and developed $\left(0.05 \%\right.$ DAB, $0.01 \% \mathrm{H}_{2} \mathrm{O}_{2}$ in PBS or with $0.7 \%$ nickel ammonium sulfate added for nickel intensification). The sections were mounted on slides, dehydrated with a series of alcohols, xylene, and embedded in DPX. TH-labeled sections were counterstained with neutral red before dehydration and embedding. For immunostaining with the $\alpha$ Enhb antibody, brains were fixed in Zamboni's solution (4\% paraformaldehyde, $100 \mathrm{mM}$ PB, $15 \%$ saturated picric acid) and then further treated as described above.

The fluorescent double labeling using $\alpha$ Enhb and anti-TH (both antibodies raised in rabbits) was performed by labeling first one, using a biotinylated anti-rabbit antibody and streptavidin-FITC, and then the other using an anti-rabbit TRITC. Labeling of the first primary antibody by the second secondary antibody was negligible and easy to differentiate because the $\alpha$ Enhb antigens, En- 1 and En-2, are localized to the nucleus, whereas $\mathrm{TH}$ is localized to the cytoplasm. In the case of double labeling for the $\beta$-gal reporter and $\mathrm{TH}$, a goat anti- $\beta$-gal, a rabbit anti-TH, and species-specific secondary antibodies were used. After the staining procedure, the sections were mounted on slides and embedded in glycerol-1,4-diazabicyclo-[2.2.2] octane.

Immunohistochemistry on whole mounts. Embryos were fixed in 4\% paraformaldehyde and stained as described (Simon et al., 1994) using the same antibody concentrations as above. Brains were isolated after fixation but before staining to facilitate penetration of the antibodies. After staining, the ventral brainstem was dissected and flat mounted in glycerol/PBS (9:1).

$R N A$ in situ hybridization. In situ detection of En-1 and En-2 on $20 \mu \mathrm{m}$ fresh-frozen sections of mouse brain was performed according to a previously described method (Goulding et al., 1994). The $\mathrm{S}^{35}$ antisense probes corresponded to regions described by Davis et al. (1988). After hybridization, sections were dipped in NTB 2 emulsion (Eastman Kodak, Rochester, NY), developed after 3-5 weeks, and counterstained.

Terminal deoxynucleotidyl transferase-mediated biotinylated UTP nick end labeling. Apoptotic cell nuclei were detected with the In Situ Cell Death Detection Kit (catalog \#1 684 809; Boehringer Mannheim, Indianapolis, IN) on either section or on isolated whole-mount embryo brains. Embryos were fixed in $4 \%$ paraformaldehyde, cryoprotected with sucrose, and cut with a freezing microtome. Sections were treated for 10 min with proteinase $\mathrm{K}$ and then stained according to the kit. The whole-mount brains were obtained by isolating the neural tubes of living embryos, which then were fixed in $4 \%$ paraformaldehyde. The specimens were dehydrated briefly and rehydrated with a series of methanol/PBS, $0.1 \%$ Tween 20 (PBT), and incubated for $10 \mathrm{~min}$ in $10 \mu \mathrm{g} / \mathrm{ml}$ proteinase $\mathrm{K}$ in PBT. After a brief wash with PBT, the specimens were incubated in the reaction mixture of the kit overnight at $4^{\circ} \mathrm{C}$. The reaction buffer was exchanged, and specimens were incubated again for $3 \mathrm{hr}$ at $37^{\circ} \mathrm{C}$. The specimens were then washed several times with PBT, $1 \mathrm{hr}$ each time, and incubated with alkaline phosphatase-coupled anti-fluorescein antibody in PBT (1:5000) overnight at $4^{\circ} \mathrm{C}$. The specimens were then washed several times with PBT, $1 \mathrm{hr}$ each time, followed by two washes with $100 \mathrm{~mm}$ Tris, $\mathrm{pH} 9.5,100 \mathrm{~mm} \mathrm{NaCl}, 50 \mathrm{~mm} \mathrm{MgCl}_{2}$, and developed with NBT/ BCIP (Boehringer Mannheim).

Image processing. All images were photographed on a Nikon Microphot-FX microscope. The film was digitized using a Nikon LS1000 scanner, and figures were assembled using Adobe Photoshop. Some images were photographed with a $2.5 \times$ lens and montaged digitally.

\section{RESULTS}

\section{$E n-1$ and En-2 are expressed in the substantia nigra and ventral tegmentum in neonatal mice}

In situ hybridizations were done on sagittal sections of postnatal day $(\mathrm{P}) 0$ mouse brains to determine the expression of the murine engrailed genes in the midbrain and hindbrain, focusing on the dopaminergic nuclei in the ventral midbrain (German and Manaye, 1993). In the ventral midbrain, $E n-1$ and $E n-2$ have largely overlapping patterns of expression (Fig. $1 A, B$ ) and are coincidentally expressed in the $\mathrm{SN}$, the $\mathrm{VT}$, and the periaqueductal central gray. Within the SN and VT, En-1 is expressed more or less throughout the nuclei at relatively high levels, whereas En-2 appears to be expressed highly in a small proportion of cells and at lower levels in the remainder. In the anterior hindbrain, En-1 expression is largely limited to the superior olive, whereas $E n-2$ is not expressed at detectable levels. Dorsally, we found that En-1 and $E n-2$ have overlapping patterns of expression, most notably the inferior colliculus and the cerebellum (Fig. 1 $A, B$ ), as reported previously (Davis and Joyner, 1988; Davis et al., 1988).

To determine whether SN and VT dopaminergic neurons express engrailed, as suggested by our findings using in situ hybridization, we performed antibody double labeling experiments. Engrailed expression was revealed with either the $\alpha$ Enhb antibody, which recognizes both En-1 and En-2 proteins (Davis and Joyner, 1988) or an antibody against $\beta$-gal to detect the tau- $\beta$-gal product of the tau-LacZ reporter in the En-1/tau-LacZ mice (Saueressig et al., 1999). Because the tau- $\beta$-gal protein integrates 

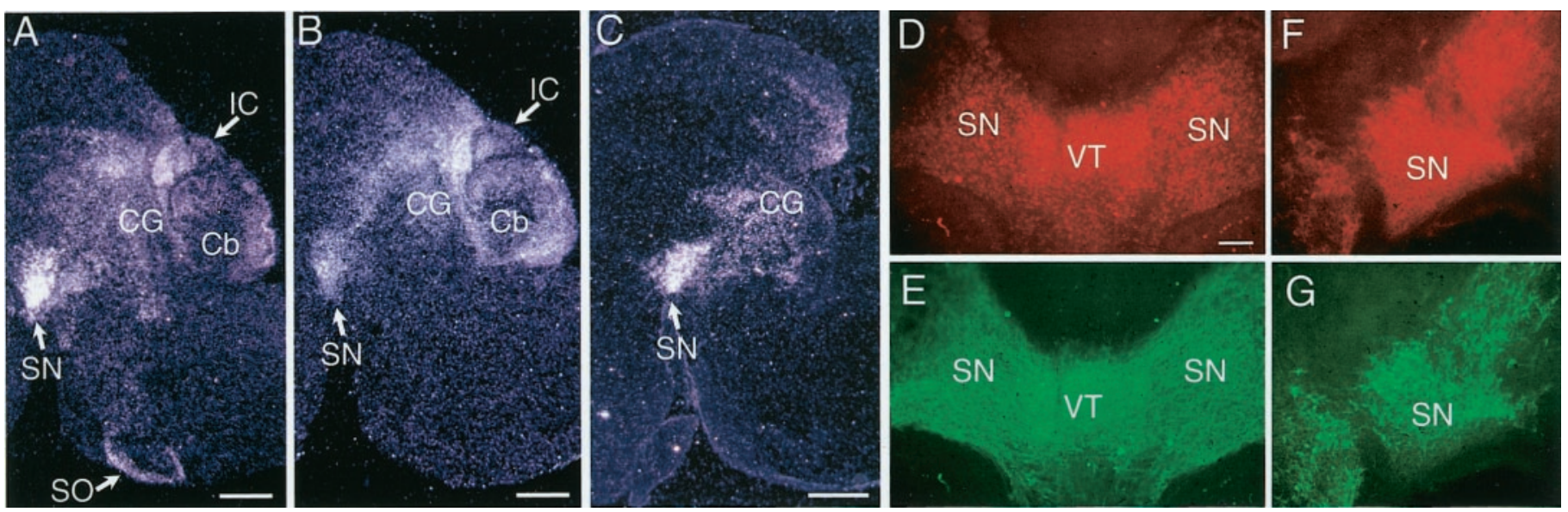

Figure 1. Dopaminergic neurons in the ventral midbrain of neonatal mice express En-1 and En-2. In situ hybridization $(A-C)$ and double immunolabeling $(D-G)$ of sagittal $(A-C, F, G)$ and coronal $(D, E)$ sections of $\mathrm{P} 0$ mouse brains. $A, B$, Adjacent sections of a P0 wild-type mouse hybridized with riboprobes against $E n-1(A)$ and $E n-2(B)$ reveal the distribution of engrailed transcripts in the substantia nigra $(S N)$, periaqueductal central gray $(C G)$, inferior colliculus $(I C)$, and cerebellum $(C b)$. En-1 is expressed at high levels throughout the ventral tegmentum $(V T)$ and SN, whereas En-2 is expressed at relatively high levels by only a small subset of cells and at much lower levels in most cells. En-1 is also expressed in a subpopulation of cells in the superior olive $(S O)$, a hindbrain nucleus. $C$, A P0 En-1 $1^{-/-}$mutant hybridized with riboprobes against En-2. In En-1 $1^{-/-}$mutants, En-2 expression appears to be upregulated and expressed at relatively high levels throughout the $\mathrm{SN}$ and VT. $D, E$, Double immunohistochemistry on a coronal section of a P0 wild-type mouse brain using the $\alpha$ Enhb antibody, which recognizes both En-1 and En-2 proteins $(D$, red), and an antibody against TH (E, green). Engrailed and TH proteins are coexpressed in dopaminergic neurons of the SN and VT. Engrailed protein is located in the nuclei, whereas TH is located in the cell somata and their axonal processes. $F, G$, A lateral section of P0 mouse brain heterozygous for En-1/tau-LacZ (En-1 $\left.{ }^{+/ t L Z}\right)$, double-labeled with antibodies against $\beta$-gal, the protein product of $L a c Z(F$, red $)$ and TH $(G$, green $)$. TH and the $\beta$-gal reporter for En-1/tau-LacZ are coexpressed in the somata and axons of the midbrain dopaminergic neurons. For the sagittal sections, rostral is to the left, and dorsal to the top. For the coronal sections, dorsal is to the top, and the midline is in the middle of the image. Scale bars: $A-C, 300 \mu \mathrm{m} ; D-G, 100 \mu \mathrm{m}$.

into the microtubule network, it effectively labels the cell body and axonal processes of En-1-expressing neurons (Callahan and Thomas, 1994; Mombaerts et al., 1996). Dopaminergic neurons were detected immunohistochemically using an antibody against $\mathrm{TH}$, a key enzyme in the synthetic pathway of dopamine. Immunostaining of P0 wild type with $\alpha$ Enhb reveals the nuclear distribution of engrailed protein in the ventral midbrain (Fig. 1D). Immunostaining the same section with the TH antibody labels the corresponding cell bodies (Fig. 1E), confirming that dopaminergic neurons of the $\mathrm{SN}$ and VT express engrailed. Because the $\alpha$ Enhb antibody recognizes both En-1 and En-2 proteins, we performed additional doublelabeling studies to determine whether midbrain dopaminergic neurons express both proteins, as suggested by our in situ data (Fig. $1 A, B)$. Double-immunolabeling of sections from $\mathrm{P} 0$ heterozygous En-1/tau-LacZ mutant brains with antibodies against $\beta$-gal and TH reveals an identical distribution (Fig. $1 F, G$ ), demonstrating that the dopaminergic neurons express En-1. In addition, doubleimmunolabeling of sections from $\mathrm{P} 0 \mathrm{En}-1^{-/-}$mice with the $\alpha$ Enhb and $\mathrm{TH}$ antibodies shows that En-2 protein is also expressed by the midbrain dopaminergic neurons (data not shown) (Fig. 1C).

\section{$E n-1$ and $E n-2$ are required for the development of midbrain dopaminergic neurons}

To determine whether the engrailed genes are required for the development of the dopaminergic neurons of the SN and VT, we analyzed En-1 (Wurst et al., 1994) and En-2 (Joyner et al., 1991; Millen et al., 1994) mutant mice. In situ hybridization analysis of P0 En-1 mutants shows that the basic expression pattern of En-2 in the ventral midbrain, including expression in the $\mathrm{SN}$ and VT, is similar to that described above in wild-type mice, with the exception that En-2 appears to be highly expressed throughout the SN (Fig. 1C), in contrast to wild type where it is highly expressed in only a proportion of SN neurons (Fig. 1B). This finding suggests that the normal coincident expression patterns of En-1 and En-2 in these nuclei do not depend on one another, although the absolute level of expression may. Therefore, because $E n-2$ can functionally replace $E n-1$ (Hanks et al., 1995), and likely vice versa, we first focused our analyses on mice deficient for both En-1 and En-2.

Dense clusters of dopaminergic neurons comprising the SN and VT are readily detected in sections of wild-type P0 mouse brains using the $\mathrm{TH}$ antibody (Fig. $2 A, A^{\prime}$ ). In contrast, in engrailed double mutants $\left(E n-1^{-/} / E n-2^{-/-}\right)$, dopaminergic neurons of the SN and VT are completely absent (Fig. 2B, $B^{\prime}$ ). Dopaminergic cell groups located outside of the expression domains of En-1 and En-2, for example the dorsomedial hypothalamic nucleus, remain detectable and appear normal in engrailed double mutants. These findings indicate that targeted deletion of both En-1 and En-2 results in the selective loss of dopaminergic neurons in the SN and VT.

\section{$E n-1$ and En-2 compensate for each other in the development of dopaminergic neurons}

To assess whether En-1 and En-2 can compensate for the targeted deletion of one another, we analyzed mutant mice null for either $E n-1$ or $E n-2$. The SN and VT in the $E n-2^{-1-}$ mice resemble wild type (data not shown), as expected on the basis of the integrity of dorsal structures in En-2 ${ }^{-/-}$mice (Joyner et al., 1991; Millen et al., 1994). In contrast, because several major dorsal structures are absent or substantially diminished in En-1 ${ }^{-1-}$ mice (Wurst et al., 1994), we anticipated a comparable reduction in the SN and VT. However, the distribution and packing density of the dopaminergic cells of the $\mathrm{SN}$ in $E n-1^{-1-}$ mice (Fig. $2 C^{\prime}$ ) appears similar to that in wild-type mice (Fig. $2 A^{\prime}$ ). The only difference that we observed is that the dopaminergic cells of the VT appear more loosely arranged in En-1 mutants than in wild-type mice (Fig. 

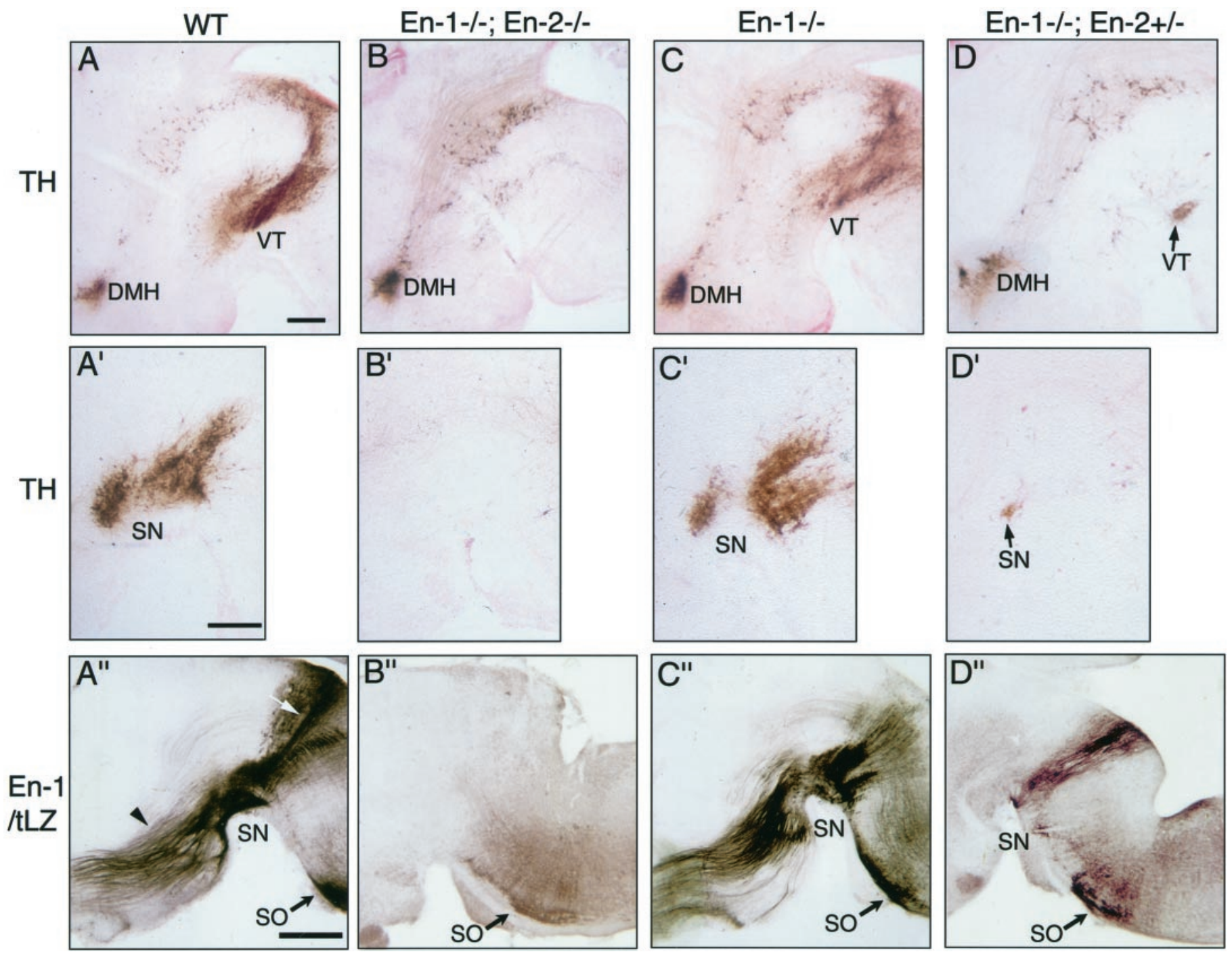

Figure 2. Midbrain dopaminergic neurons require En-1 and En-2 for their survival in a gene dose-dependent manner. Immunohistochemistry on sagittal sections of $\mathrm{P} 0$ mouse brains using antibodies against tyrosine hydroxylase $(T H)$ to identify dopaminergic neurons $\left(A, A^{\prime}, B, B^{\prime}, C, C^{\prime}, D, D^{\prime}\right)$, or against $\beta$-gal (A", B", $\left.\mathrm{C}^{\prime \prime}, \mathrm{D}^{\prime \prime}\right)$ to identify En-1/tau-LacZ (En-1/tLZ)-expressing neurons in the ventral midbrain of wild-type $(W T)$ mice $\left(A-A^{\prime \prime}\right)$, engrailed double mutants $\left(E n-1^{-1-} \mid E n-2^{-/-}\right)\left(B-B^{\prime \prime}\right), E n-1^{-/-}$mutants $\left(C-C^{\prime \prime}\right)$, and $E n-1^{-/-} / E n-2^{+/-}$mutants $\left(D--D^{\prime \prime}\right)$. In $A^{\prime \prime}-D^{\prime \prime}$, mice with the En-1/tau-LacZ $(E n-1 / t L Z)$ reporter construct were used. Therefore, in $A^{\prime \prime}-D^{\prime \prime}$, wild type is actually $E n-1^{+/ \mathrm{LLZ}}$, and $E n-1^{-/-}$is actually $E n-1^{\text {tLZ/tLZ }} . A-A^{\prime \prime}$, Wild type. TH immunostaining reveals the normal distribution of the midbrain dopaminergic neurons. In the midline $(A)$, the neurons of the ventral tegmentum $(V T)$ are labeled, and in a more lateral section $\left(A^{\prime}\right)$, those of the substantia nigra $(S N)$ are labeled. The $\beta$-gal immunostaining of a section from an $E n-1^{+/ t L Z}$ reveals a similar labeling pattern as TH for both the VT and SN. In addition, SN axons projecting rostrally to the forebrain (A", arrowhead) and En-1-expressing radial glia (white arrow) are labeled. $D M H$, dorsomedial hypothalamic nucleus. $B-B^{\prime \prime}$, Engrailed double mutant. Dopaminergic neurons of the SN and VT are not detected by immunostaining with either TH $\left(B, B^{\prime}\right)$ or $\beta$-gal $\left(B^{\prime \prime}\right)$. However, dopaminergic neurons of the $D M H($ a diencephalic nucleus) remain TH-positive $\left(B-B^{\prime \prime}\right)$, indicating that their dopaminergic phenotype does not depend on En-1 or En-2. In addition, neurons in the superior olive $(S O)$ that normally express En-1 are marked by the En-1/tau-LacZ reporter in engrailed double mutants $\left(B^{\prime \prime}\right)$, as well as in the other mutant genotypes examined $\left(C^{\prime \prime}, D^{\prime \prime}\right)$, indicating that En-1 expression is not auto-regulated. $C-C^{\prime \prime}, E n-1^{-/-}$mutant. Distribution of the dopaminergic neurons appears normal with the exception of those located in the VT that appear more loosely clustered (compare $C$ with $A$ ). $D-D^{\prime \prime}, E n-1^{-1-} / E n-2^{+/-}$mice. The dopaminergic neurons of the SN and VT are reduced to a small cluster of cells in the ventral midbrain, demonstrating an engrailed gene dose-dependent effect on the development of these neurons. Rostral to the left, dorsal to the top. Scale bars, $200 \mu \mathrm{m}$.

2, compare $A, C)$. The relatively normal appearance of the $\mathrm{SN}$ and VT in engrailed single mutants, compared with their absence in engrailed double mutants, indicates that En-1 and En-2 compensate substantially, if not entirely, for the loss of one another in regulating the proper development of these nuclei.

\section{En-1 and En-2 act in a gene dose-dependent manner}

To determine whether En-1 and En-2 act in a gene dosedependent manner, we analyzed mutant mice null for one engrailed gene and heterozygous for the other. In $E n-1^{-/-} / E n-2^{+/-}$ mice, the SN and VT are substantially diminished, compared with engrailed single mutants or wild-type mice, being reduced to a small cluster of dopaminergic neurons close to the ventral surface of the midbrain (Fig. 2D, $D^{\prime}$ ). In contrast to these substantial defects in $E n-1^{-/-} / E n-2^{+/-}$mice, in $E n-1^{+/-} / E n-2^{-/-}$mice the $\mathrm{SN}$ and VT are essentially identical to the wild type (data not shown). Thus, with respect to the dopaminergic neurons of the $\mathrm{SN}$ and VT, in mice in which only one of the four engrailed allelesis present, one En-1 allele is sufficient to produce a phenotype that resembles wild type, but one En-2 allele is not. 


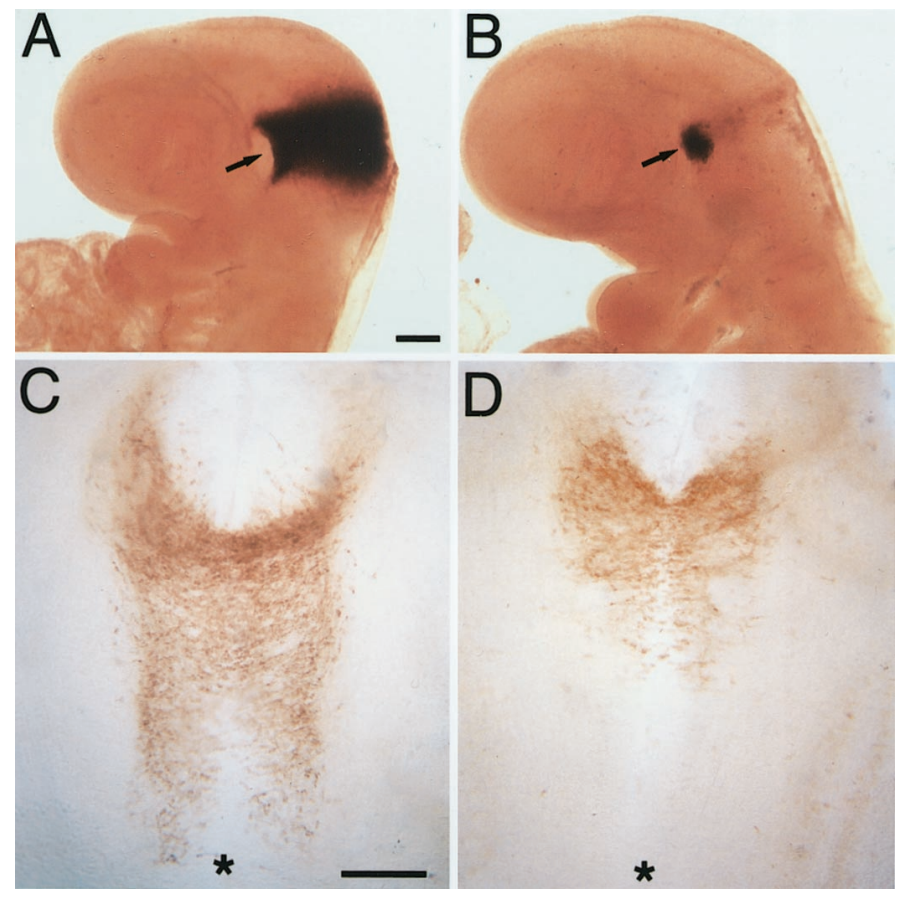

Figure 3. Dopaminergic neurons of the ventral midbrain are generated in engrailed double mutants. Whole-mount immunohistochemistry of E9 $(A, B)$ and $\mathrm{E} 11(C, D)$ embryos using antibodies against $\beta$-gal and $\mathrm{TH}$, respectively. $A$, Heterozygous E9 embryo $\left(E n-1^{+ \text {tLZZ }}\right)$. $\beta$-gal immunostaining reveals the normal distribution of En-1. En-1 is expressed rostral and caudal to the isthmus (arrow) spanning the entire neuraxis from dorsal to ventral. $B$, In the E9 engrailed double mutant embryo

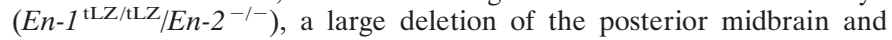
anterior hindbrain is apparent. $\beta$-gal immunostaining is only detected in a small domain in the ventral neural tube (arrow), likely corresponding to the precursor cells of the dopaminergic neurons. $C$, E11 wild-type flat mount of ventral midbrain immunostained for TH. The immunostaining reveals the bilateral distribution of dopaminergic neurons adjacent to the floor plate (asterisk). D, E11 engrailed double mutants $\left(E n-1^{-1-} /\right.$ $\left.E n-2^{-1-}\right)$. A cluster of dopaminergic neurons is detected by TH immunostaining, although it is smaller than that observed in wild-type or heterozygous mice. These neurons disappear in engrailed double mutants over the next few days of development. Rostral is to the top. Scale bars, $100 \mu \mathrm{m}$.

\section{Midbrain dopaminergic neurons are absent rather than} phenotypically defective in engrailed double mutants

One of three possible explanations could account for our inability to detect in engrailed double mutants the dopaminergic neurons that would normally form the SN and VT: they are present but defective in expressing their dopaminergic phenotype, they are not generated, or they are generated but disappear later. To address this issue, we used the En-1/tau-LacZ mice in which En-1 is replaced with tau-LacZ, creating a null En-1 allele (termed $E n-1^{\text {tLZ }}$ ) (see Materials and Methods) (Saueressig et al., 1999). In these mice, the tau- $\beta$-gal protein encoded by tau-LacZ is a cell autonomous marker specific for En-1-expressing neurons, and it labels their cell bodies and axonal processes. With respect to the $\mathrm{SN}$ and VT, the distribution of tau- $\beta$-gal protein is the same as the distribution of $\mathrm{TH}$ protein in each of the six En-1/En-2 genotype combinations analyzed in this study (Fig. 2; data not shown) (for determining six genotypes, the $E n-1^{\text {tLZ }}$ allele is considered equivalent to the En-1 ${ }^{-/-}$allele), including the engrailed double mutants $\left(E n-1^{\mathrm{tLZ} / \mathrm{tLZ}} / E n-2^{-/-}\right)$in which no tau$\beta$-gal-positive or TH-positive neurons are detected in the ventral midbrain (Fig. 2B-- $B^{\prime \prime}$ ). However, En-1-expressing cells in the superior olive are present in all genotypes analyzed, including the engrailed double mutant, indicating that En-1 is not positively autoregulated (Saueressig et al., 1999). These findings strongly suggest that the lack of detection of the midbrain dopaminergic neurons is attributable to their absence rather than their failure to express a dopaminergic phenotype.

\section{Midbrain dopaminergic neurons are generated in engrailed double mutants}

The absence of dopaminergic neurons of the SN and VT in engrailed double mutants could be caused by an early event such as the failure of these cells to be generated or to a later event such as the loss of these cells after they have differentiated and started to express TH. At embryonic day (E) $8, E n-1$ and $E n-2$ are broadly expressed in the neuroepithelium of the posterior midbrain and anterior hindbrain and appear to be involved in specifying these fields (Wurst et al., 1994). If the deletion of En-1 and En-2 results in a respecification or diminished proliferation of the neuroepithelial cells that normally would generate the dopaminergic neurons or their precursors, the dopaminergic neurons would not be detectable at any stage of development. If the defect observed in engrailed double mutants is caused by a later event, we would expect that the dopaminergic neurons could be detected over at least a brief embryonic period. To address these alternatives, we first determined whether any cells that normally express En-1 remain in the midbrain after the full extent of the morphological deletion becomes apparent around E9 (Wurst et al., 1994). This approach is made possible by using the En-1/tau-LacZ mice.

In hetero-homo E9 embryos $\left(E n-1^{+/ t \mathrm{LZ}} / E n-2^{-/-}\right)$, the tau$L a c Z$ domain detected by the $\beta$-gal antibody coincides exactly with the normal En-1 expression domain in the wild type (Fig. $3 A$ ). In engrailed double mutant embryos at the same age, this domain is substantially reduced to a small group of tau-LacZ positive cells in the ventral neural tube. This zone corresponds with the area in which the dopaminergic neurons are induced (Ye et al., 1998) slightly later. Because the precursor cells giving rise to midbrain dopaminergic neurons are present in engrailed double mutants, we used TH immunostaining to determine whether neurons of the SN and VT are generated and express their dopaminergic phenotype. At E11, in wild-type and hetero-homo $\left(E n-1^{+/-} / E n-2^{-/-}\right)$mutants, a distinct ventral domain of THpositive cells is present in the midbrain (Fig. $3 C$ ). These cells will later form the SN and VT (Voorn et al., 1988; Lieb et al., 1996). In engrailed double mutants $\left(E n-1^{-/} / E n-2^{-/-}\right)$, this ventral domain of TH-positive cells is present at this age (Fig. 3D), although it is smaller than that observed in $E n-1^{+/-} / E n-2^{-/-}$littermates or wild-type mice. By E14, these TH-positive cells are no longer detectable in engrailed double mutants (data not shown; for P0, see Fig. $3 B$ ). These findings indicate that neurons of the dopaminergic phenotype are generated in the ventral midbrain of engrailed double mutants, but they disappear soon thereafter.

Our findings suggest that in engrailed double mutants, midbrain dopaminergic neurons are generated but die over a brief time window of embryonic development. Because most embryonic cell death is apoptotic and apoptotic cell death can often be recognized by methods detecting chromosomal fragmentation (Compton, 1992), we used a terminal deoxynucleotidyl transferase-mediated biotinylated UTP nick end labeling (TUNEL) technique to identify apoptotic cell nuclei in the ventral midbrain in wild-type mice and engrailed double mutants at E12 and E13, ages when the midbrain dopaminergic neurons can still be identified in the mutants but are reduced in number 
compared with wild type. We found no TUNEL-positive cells in either wild-type or engrailed double mutants in the region of the ventral midbrain in which the dopaminergic neurons are located, whereas unrelated neuronal populations known to undergo apoptotic cell death at these ages, such as the trigeminal ganglia (Davies and Lumsden, 1984), show large numbers of TUNELpositive cells (data not shown). These findings suggest that if the midbrain dopaminergic neurons die via apoptosis, their turnover rate or some other feature precludes their detection with the TUNEL method.

\section{Engrailed expression in midbrain dopaminergic neurons is maintained in the adult}

Because the degeneration of dopaminergic neurons in the SN and VT is implicated in PD, we investigated whether engrailed expression observed during embryogenesis and in neonates is maintained into the adult. We determined whether $E n-1$ and $E n-2$ are expressed by the dopaminergic neurons of the $\mathrm{SN}$ and VT of adult mice by immunostaining for $\beta$-gal in mice heterozygous for En-1/tau-LacZ or by in situ hybridization. The SN and VT are strongly positive for tau- $\beta$-gal protein in adult $E n-1^{+/ t L Z}$ mice (Fig. $4 A$ ). In addition, the axonal projections of the $\mathrm{SN}$ and $\mathrm{VT}$ to their major targets, such as the striatum, nucleus accumbens, and the olfactory tubercle (Voorn et al., 1988; Paxinos et al., 1994), are also immunostained for tau- $\beta$-gal protein. This overall pattern of $\beta$-gal immunostaining is identical to that for TH immunostaining (Fig. 4B), demonstrating that the dopaminergic neurons of the SN and VT continue to express En-1 in the adult. In situ hybridization confirms the En-1 expression data obtained with the En-1/tau-LacZ mice and shows that En-2 is also expressed in the SN and VT (Fig. 4C,D). However, as during development (see above), En-1 is expressed at high levels throughout the SN and VT, whereas En-2 appears to be expressed highly in only a subset of cells in these nuclei and at considerably lower levels in the remainder. Thus En-1 and En-2 are expressed in the dopaminergic neurons of the $\mathrm{SN}$ and VT from early embryogenesis into adult, although their relative levels of expression appear to differ.

\section{$\alpha$-Synuclein may be regulated by engrailed}

Our findings in mice that En-1 and En-2 are required for the survival of dopaminergic neurons of the SN and VT and are expressed by these neurons in the adult suggest a link between these genes and PD. Thus, we investigated whether the engrailed genes influence the expression of $\alpha$-synuclein, which is expressed by the midbrain dopaminergic neurons (Maroteaux et al., 1988; Maroteaux and Scheller, 1991) and has been genetically linked to PD in humans (Polymeropoulos et al., 1997). We isolated mouse $\alpha$-synuclein cDNA and performed an in situ hybridization analysis of its expression at late E12, an age when midbrain dopaminergic neurons have been generated and can be defined by TH expression but have yet to be lost in engrailed double mutants. We found that in wild-type mice, $\alpha$-synuclein expression (Fig. $5 A$ ) colocalizes with midbrain dopaminergic neurons identified by in situ hybridization for TH (Fig. 5B). Expression of $\alpha$-synuclein by midbrain dopaminergic neurons is diminished in $E n-1$ null mice (Fig. 5, compare $C, D$ ) and absent in engrailed double mutants (Fig. 5, compare $C, E$ ), although the TH-positive neurons are still present (Fig. 3). These findings indicate that En-1 and En-2 regulate the expression of $\alpha$-synuclein in ventral midbrain dopaminergic neurons.

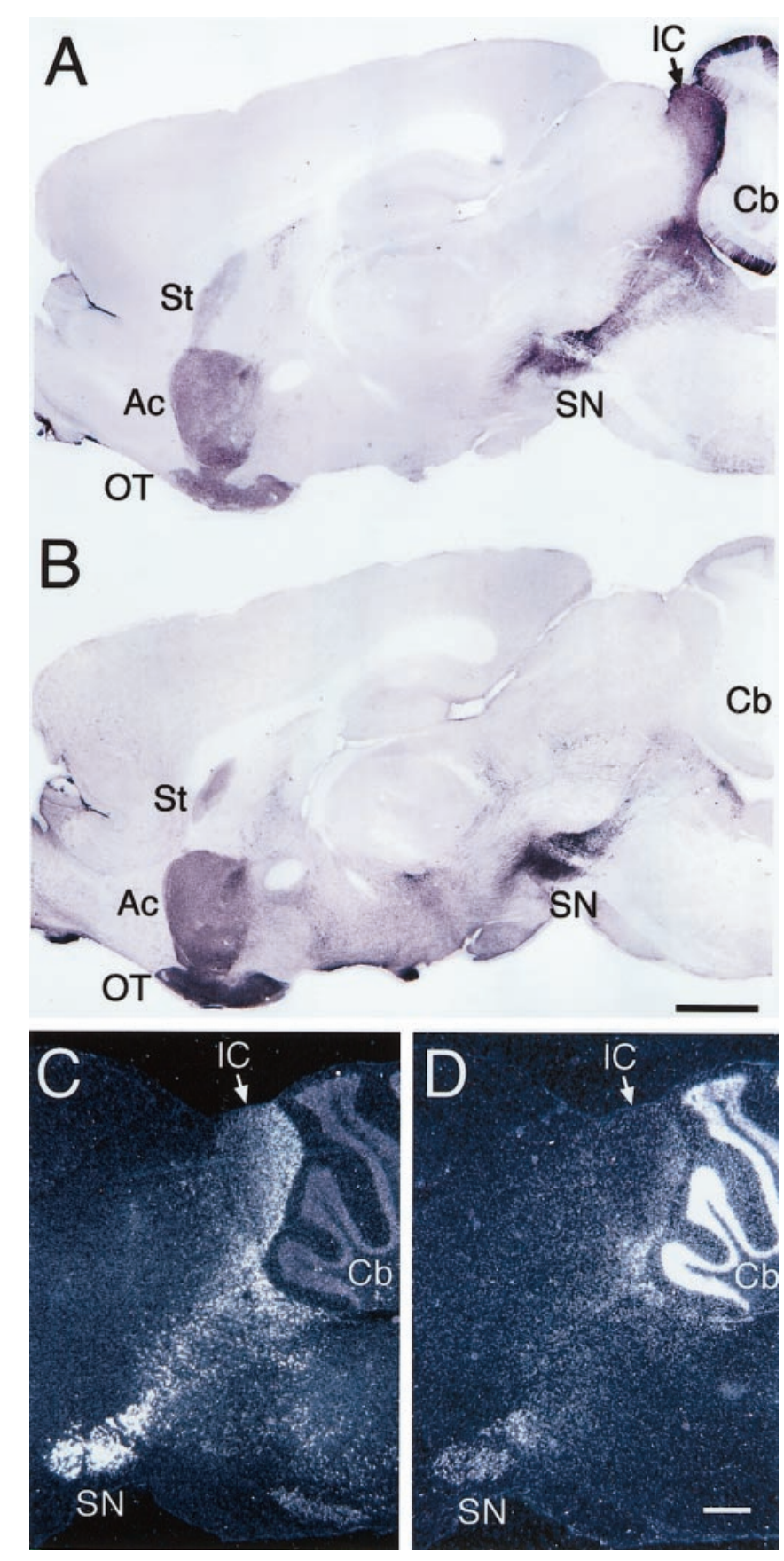

Figure 4. Dopaminergic neurons of the substantia nigra express En-1 and $E n-2$ in adult mice. Immunohistochemistry $(A, B)$ and in situ hybridization $(C, D)$ on sagittal sections of adult mouse brain. $A$, Immunostaining for $\beta$-gal on an $E n-1^{+/ t \mathrm{LZ}}$ brain section reveals $E n-1$ expression domains in the midbrain: the substantia nigra $(S N)$, inferior colliculus $(I C)$, parts of the periaqueductal central gray, and a subset of the Bergmann glia in the cerebellum $(\mathrm{Cb})$. Because of the cytoplasmic localization of the tau- $\beta$-gal reporter protein, the axonal projection of the $\mathrm{SN}-\mathrm{VT}$ dopaminergic neurons to the striatum $(S t)$, the nucleus accumbens $(A c)$, and the olfactory tubercle $(O T)$ is also labeled by the $\beta$-gal antibody. $B$, Immunostaining for $\mathrm{TH}$ on a section adjacent to the one shown in $A$, revealing the distribution of dopaminergic neurons in the SN and their axonal terminations (St, Ac, and OT). The similar distribution of $\beta$-gal and TH in $A$ and $B$ demonstrates that dopaminergic neurons of the SN express $E n-1$ in the adult. $C$, $D$, Adjacent sections hybridized with $\mathrm{S}^{35}$-labeled riboprobes against $E n-1(C)$ or $E n-2(D)$. En-1 is expressed at high levels throughout the SN. In contrast, En-2 is expressed at high levels in only a small subset of cells in the SN and at much lower level in the majority of SN cells. Rostral is to the left, dorsal to the top. Scale bars: $A, B, 1 \mathrm{~mm} ; C, D, 500 \mu \mathrm{m}$. 
Figure 5. The engrailed genes influence the expression of $\alpha$-synuclein. Whole-mount in situ hybridization using riboprobes against mouse $\alpha$-synuclein $(A, C, D, E)$ and mouse $\mathrm{TH}(B)$ on E12 brains from wild-type mice $(A-C), E n-1^{-1-}$ mutants $(D)$, and engrailed double mutants $\left(E n-1^{-1-} \mid E n-2^{-1-}\right)$ (E). $A, B$, Whole-mount in situ of a wildtype brain showing a view of the ventral midbrain (dorsal midbrain is removed). $\alpha$-synuclein $(A)$ and $T H(B)$ transcripts are largely colocalized in bilateral domains ( $a r-$ rows) adjacent to the midline (asterisks) in the anterior ventral midbrain. The $T H$ expression domain is the larger of the two and is composed of the dopaminergic neurons that give rise to both the $\mathrm{SN}$ and VT, whereas the $\alpha$-synuclein expression domain likely only marks developing SN neurons at this age. $C$, Wild-type flat mount of the ventral midbrain shows the bilateral $\alpha$-synuclein expression domain (arrow) adjacent to the midline. $D, E n-1^{-/-}$flat mount of the ventral midbrain shows a domain of $\alpha$-synuclein expression (arrow) at the same location as in the wild type, but the expression level is greatly reduced. $E$, Engrailed double mutant, $\alpha$-synuclein is not detected in its normal expression domain adjacent to the midline, although TH and engrailed positive dopaminergic neurons are still present at this age (Fig. 3D). Note that the more ante-
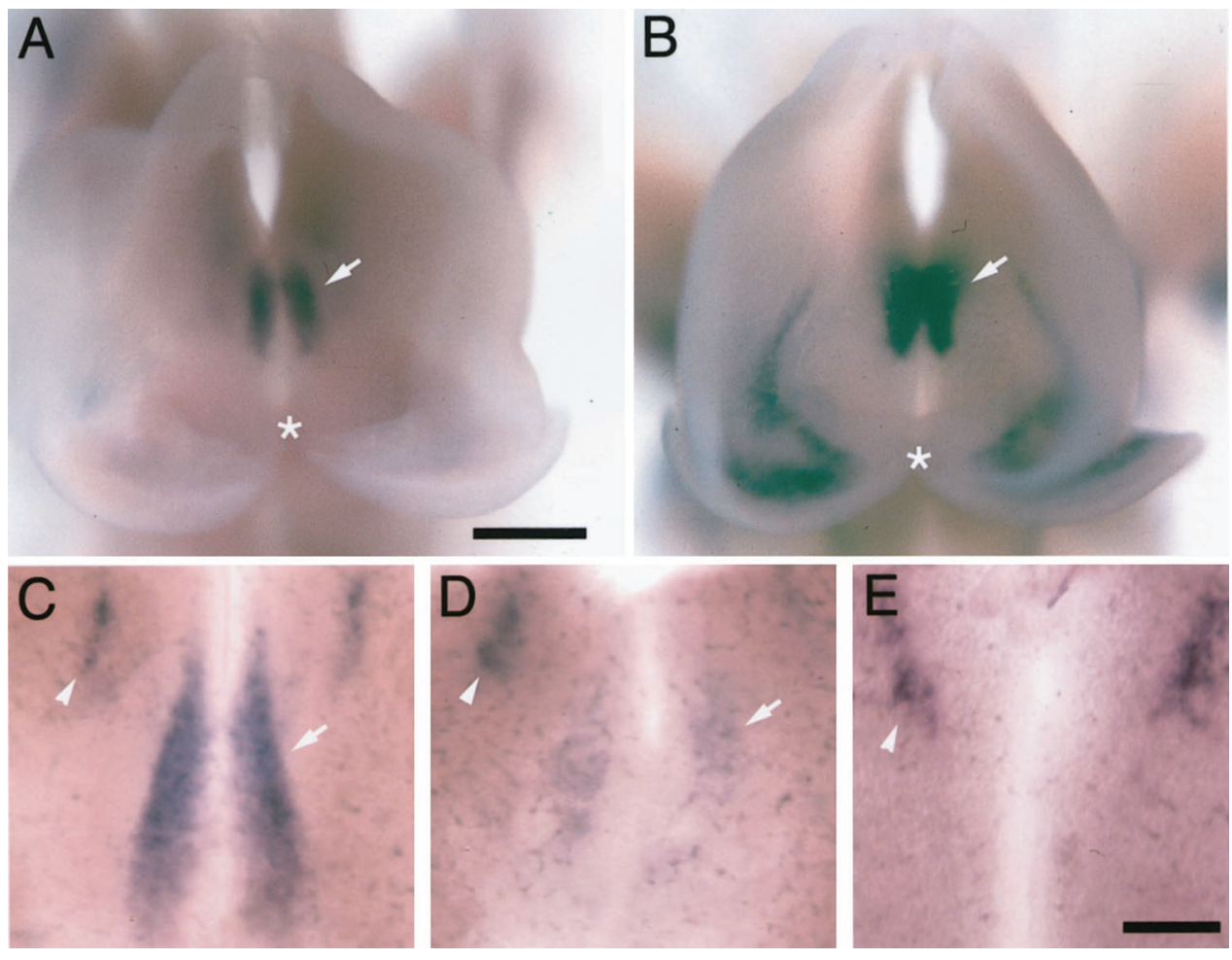

rior and lateral domains of $\alpha$-synuclein expression in the diencephalon $(C-E$, arrowheads $)$ is unaffected in all three genotypes, indicating that the diminished $\alpha$-synuclein expression in the engrailed mutants is attributable to the lack of engrailed expression in the SN and not to a developmental delay.

\section{DISCUSSION}

We show that $E n-1$ and $E n-2$ are required in mice for the survival of dopaminergic neurons of the SN and VT in a gene dosedependent manner. These neurons are generated and differentiate their dopaminergic phenotype independent of engrailed expression but are lost soon thereafter if both En-1 and En-2 are absent. The proportion that survives depends on the En-1/En-2 genotype. Furthermore, we show that the expression of $\alpha$-synuclein is dependent on engrailed. These findings raise issues regarding the mechanisms of action of En-1 and En-2 in controlling the developmental fate and survival of these neurons and a potential link between engrailed and PD.

\section{Mechanisms underlying the loss of midbrain dopaminergic neurons}

Our analyses using two independent markers, TH and En-1/tauLacZ, show that dopaminergic neurons of the SN and VT are generated in engrailed double mutants but cannot be detected a few days later. This finding indicates that engrailed is not required for $\mathrm{TH}$ expression and therefore is the dopaminergic phenotype of SN and VT neurons, which is consistent with the fact that the expression domains of neither En-1 nor En-2 coincide elsewhere in the CNS with the location of dopaminergic neurons (Davis and Joyner, 1988; Davis et al., 1988). It is likely that the ventral midbrain dopaminergic neurons die shortly after they are generated in engrailed double mutants, because the alternative, that they are present but no longer detectable, would demand that the initiation of expression of TH and En-1/tau-LacZ does not require either $E n-1$ or $E n-2$, but their maintained expression does. Such a late onset of regulation of $E n-1$ by engrailed is rendered further unlikely by our finding that En-1-expressing neurons in the superior olive appear unaffected and are marked by the tau- $\beta$-gal reporter in engrailed double mutants. At least in these cells, the maintained expression of En-1/tau-LacZ is engrailed independent.

Thus, the evidence indicates that the midbrain dopaminergic cells require engrailed for their survival. Their survival may depend on signals or trophic support provided by other cells that are normally present in the ventral midbrain of wild-type mice and single null mutants for either En-1 or En-2 but are missing in engrailed double mutants. This explanation is consistent with the more substantial truncation of the midbrain-anterior hindbrain in engrailed double mutants than in either single mutant (Millen et al., 1994; Wurst et al., 1994). Alternatively, the loss of the midbrain dopaminergic neurons in engrailed double mutants could be caused by a defect autonomous to these neurons. For example, essential housekeeping genes or components of essential signaling pathways, including receptors for required trophic factors, might be controlled by engrailed and are improperly regulated in midbrain dopaminergic neurons in engrailed double mutants. Candidate receptors are Nurr1, an orphan member of the steroid-thyroid hormone receptor family, and receptors for glial cell line-derived neurotrophic factor (GDNF), a member of the TGF- $\beta$ superfamily.

TH-positive cells are not detected at embryonic or postnatal ages in the ventral midbrain of Nurr1 mutant mice (Zetterström et al., 1997). However, use of markers for afferents to the SN, substance $\mathrm{P}$, and glutamic acid decarboxylase, and the neuronspecific nuclear marker NeuN, suggests that SN neurons are present in Nurr1 mutants but do not express TH (Castillo et al., 1998), consistent with the in vitro demonstration that Nurr1 can induce TH expression in cells resembling midbrain dopaminergic neurons (Sakurada et al., 1999). It is unlikely that En-1 and En-2 regulate Nurr1 because, in engrailed double mutants, ventral 
midbrain TH-positive neurons are detected during early embryogenesis but later disappear.

GDNF, which has potent trophic effects on SN and VT dopaminergic neurons, increases their survival and differentiation in vitro (Lin et al., 1993) and prevents their loss in the adult after axotomy (Beck et al., 1995) or treatment with the neurotoxin 1-methyl-4-phenyl-1,2,3,6-tetrahydropyridine (Tomac et al., 1995), which induces a PD-like syndrome. The receptor complex for GDNF is composed of GDNFR $\alpha$ (GDNF receptor $\alpha$ ) and Ret (Jing et al., 1996; Baloh et al., 1997), which are expressed in the SN and VT of mice from at least E13.5 into adulthood (Avantaggiato et al., 1994; Nosrat et al., 1997). Regulation of GDNFR $\alpha$ and Ret by En-1 and En-2 is possible, but unlikely to be the cause for the loss of midbrain dopaminergic neurons in engrailed double mutants, because a reduction in this population is not apparent in mice with a targeted deletion of either GDNF or Ret (Schuchardt et al., 1994; Moore et al., 1996).

\section{Compensatory mechanisms of En-1 and En-2}

In contrast to the complete loss of $\mathrm{SN}$ and VT dopaminergic neurons in engrailed double mutants, mice null for either En-1 or $E n-2$ have no apparent reductions in these populations. This finding indicates that En-1 and En-2 compensate for the deletion of each other in maintaining the differentiation and survival of these neurons. Although En-2 can functionally replace $E n-1$ when knocked-in to the En-1 locus (Hanks et al., 1998), they differ substantially in their compensatory abilities to maintain ventral midbrain dopaminergic neurons; whereas a single allele of $E n-1$ is sufficient to maintain a wild type-like phenotype, a single allele of $E n-2$ is not. This difference may be attributable to En-1 expression beginning before En-2 (Davidson et al., 1988; Davis and Joyner, 1988; Davis et al., 1988; McMahon et al., 1992). More likely though, it is attributable to our finding that during development and in the adult, En-2 is expressed at high levels in only a small proportion of $\mathrm{SN}$ and VT neurons, whereas En-1 is expressed at high levels in most or all of them. We suggest that the small proportion of SN and VT neurons that normally express high levels of $E n-2$ are those that survive in the $E n-1^{-/-} / E n-2^{+/-}$ mice, whereas no loss is apparent in $E n-1^{+/-} / E n-2^{-/-}$mice because essentially all of these neurons highly express En-1.

\section{Potential effects on induction of midbrain dopaminergic neurons}

Progenitor cells in the neuroepithelium of the ventral midbrain are induced to produce dopaminergic neurons by the combined action of sonic hedgehog $(\mathrm{SHH})$ released by the floorplate at the ventral midline and FGF8 produced by anterior forebrain and the isthmus, a strip of tissue at the midbrain-hindbrain junction (Ye et al., 1998). The population of ventral midbrain dopaminergic neurons observed at E12 was reduced in every engrailed double mutant analyzed, compared with their littermates: both $E n-1^{+/+} / E n-2^{-/-}$embryos, which have a wild-type-like phenotype, and $E n-1^{-/+} / E n-2^{-/-}$embryos, which have an intermediate phenotype. One potential explanation for the reduced population at E12 in the engrailed double mutants is a temporal overlap in the loss and generation of TH-positive neurons. Alternatively, levels of SHH or FGF8 are reduced in engrailed double mutants, and fewer progenitor cells respond to these lower concentrations to produce dopaminergic neurons. Because the isthmus is absent in engrailed double mutants (present study), a lower level of FGF8 might be expected. However, the isthmus is also absent in En-1 mutants (Wurst et al., 1994), but the number of midbrain dopaminergic neurons appears unaffected. A third possibility is that engrailed double mutants have a reduced number of progenitor cells capable of producing dopaminergic neurons. Consistent with this possibility is our finding that the En-1/tau-LacZ expression domain, which includes the ventral midbrain neuroepithelium in which these progenitors are located, is substantially reduced in engrailed double mutants (Fig. $3 A, B$ ).

\section{Engrailed and Parkinson's disease}

Degeneration of SN and VT neurons and the loss of their dopaminergic innervation of the forebrain is the primary cause of the severe movement disorders associated with PD (Jenner et al., 1992; Temlett, 1996). PD, which affects 1-2\% of humans during their lifetime (Polymeropoulos et al., 1996), can be caused by genetic and epigenetic factors (Tomac et al., 1995). A link between engrailed and PD is suggested by our findings that SN and VT dopaminergic neurons require $E n-1$ and $E n-2$ for their survival, that En-1 and En-2 continue to be expressed by these neurons in the adult, and that expression of $\alpha$-synuclein, a gene on human chromosome 4 genetically linked to PD in some families (Polymeropoulos et al., 1997), is dependent on engrailed. It is unlikely, though, that both En-1 and En-2 are mutated in a given individual, especially because they are located on different chromosomes (human chromosomes 2 and 7, respectively) (Logan et al., 1989). However, because En-1 is expressed at high levels throughout the SN and VT in adult mice, whereas En-2 is expressed at high levels in only a small proportion of SN and VT neurons, a mutation in En-1 may be sufficient to promote the onset of PD. A mutation of En-1 would not necessarily affect the development of midbrain dopaminergic neurons, because as shown here, they appear unaffected even in En-1 null mice, indicating that during development $E n-2$ compensates for the lack of En-1. However, an age-related decrease in the ability of $E n-2$ to compensate for either the loss of En-1 or a change in its amount or efficacy caused by a mutation in the regulatory or coding sequences for En-1 may lead to a gradual degeneration of dopaminergic neurons in the $\mathrm{SN}$ and VT as occurs in PD. This scenario is consistent with our finding of an En-2 gene dose effect, which indicates that the number of dopaminergic neurons that survive in the absence of En-1 appears to depend on the level of En-2 expression.

Another potential link with PD is our finding suggesting that engrailed regulates $\alpha$-synuclein expression. Transgenic mice engineered to overproduce $\alpha$-synuclein, a presynaptic and nuclear protein believed to be involved in synaptic plasticity, exhibit a loss of midbrain dopaminergic neurons (Masliah et al., 2000). It is conceivable that an alteration in the binding sequence of the $\alpha$-synuclein gene for En-1 or En-2 or a mutation in either engrailed gene could lead to an increase of $\alpha$-synuclein, which may in turn lead to the degeneration of dopaminergic neurons in the ventral midbrain.

\section{REFERENCES}

Avantaggiato V, Dathan NA, Grieco M, Fabien N, Lazzaro D, Fusco A, Simeone A, Santoro M (1994) Developmental expression of the RET protooncogene. Cell Growth Differ 5:305-311.

Baloh RH, Tansey MG, Golden JP, Creedon DJ, Heuckeroth RO, Keck CL, Zimonjic DB, Popescu NC, Johnson Jr EM, Milbrandt J (1997) TrnR2, a novel receptor that mediates neurturin and GDNF signaling through Ret. Neuron 18:793-802.

Beck KD, Valverde J, Alexi T, Poulsen K, Moffat B, Vandlen RA, Rosenthal A, Hefti F (1995) Mesencephalic dopaminergic neurons protected by GDNF from axotomy-induced degeneration in the adult brain. Nature 373:339-341. 
Callahan CA, Thomas JB (1994) Tau-beta-galactosidase, an axontargeted fusion protein. Proc Natl Acad Sci USA 91:5972-5976.

Castillo SO, Baffi JD, Palkovits M, Goldstein DS, Kopin IJ, Witta J, Magnuson MA, Nikodem VM (1998) Dopamine biosynthesis is selectively abolished in substantia nigra/ventral tegmental area but not in hypothalamic neurons in mice with targeted disruption of the Nurr1 gene. Mol Cell Neurosci 11:36-46.

Compton MM (1992) A biochemical hallmark of apoptosis: internucleosomal degradation of the genome. Cancer Metastasis Rev 11:105-119.

Condron BG, Patel NH, Zinn K (1994) Engrailed controls glial/neuronal cell fate decisions at the midline of the central nervous system. Neuron 13:541-554.

Davidson D, Graham E, Sime C, Hill R (1988) A gene with sequence similarity to Drosophila engrailed is expressed during the development of the neural tube and vertebrae in the mouse. Development 104:305-316.

Davies A, Lumsden A (1984) Relation of target encounter and neuronal death to nerve growth factor responsiveness in the developing mouse trigeminal ganglion. J Comp Neurol 223:124-137.

Davis CA, Joyner AL (1988) Expression patterns of the homeo boxcontaining genes En-1 and En-2 and the proto-oncogene int-1 diverge during mouse development. Genes Dev 2:1736-1744.

Davis CA, Noble-Topham SE, Rossant J, Joyner AL (1988) Expression of the homeo box-containing gene En-2 delineates a specific region of the developing mouse brain. Genes Dev 2:361-371.

Friedman GC, O'Leary DDM (1996) Retroviral misexpression of engrailed genes in the chick optic tectum perturbs the topographic targeting of retinal axons. J Neurosci 16:5498-5509.

Gardner CA, Darnell DK, Poole SJ, Ordahl CP, Barald KF (1988) Expression of an engrailed-like gene during development of the early embryonic chick nervous system. J Neurosci Res 21:426-437.

German DC, Manaye KF (1993) Midbrain dopaminergic neurons (nuclei A8, A9, and A10): three-dimensional reconstruction in the rat. J Comp Neurol 331:297-309.

Goulding M, Lumsden A, Paquette AJ (1994) Regulation of Pax-3 expression in the dermomyotome and its role in muscle development. Development 120:957-971.

Hanks M, Wurst W, Anson-Cartwright L, Auerbach AB, Joyner AL (1995) Rescue of the En-1 mutant phenotype by replacement of En-1 with En-2. Science 269:679-682

Hanks MC, Loomis CA, Harris E, Tong CX, Anson-Cartwright L, Auerbach A, Joyner A (1998) Drosophila engrailed can substitute for mouse Engrailed1 function in mid-hindbrain, but not limb development. Development 125:4521-4530.

Itasaki N, Nakamura H (1996) A role for gradient en expression in positional specification on the optic tectum. Neuron 16:55-62.

Jenner P, Schapira AH, Marsden CD (1992) New insights into the cause of Parkinson's disease. Neurology 42:2241-2250.

Jing S, Wen D, Yu Y, Holst PL, Luo Y, Fang M, Tamir R, Antonio L, Hu Z, Cupples R, Louis JC, Hu S, Altrock BW, Fox GM (1996) GDNFinduced activation of the ret protein tyrosine kinase is mediated by GDNFR-alpha, a novel receptor for GDNF. Cell 85:1113-1124.

Joyner AL, Herrup K, Auerbach BA, Davis CA, Rossant J (1991) Subtle cerebellar phenotype in mice homozygous for a targeted deletion of the En-2 homeobox. Science 251:1239-1243.

Kornberg T (1981a) Engrailed: a gene controlling compartment and segment formation in Drosophila. Proc Natl Acad Sci USA 78:1095-1099.

Kornberg T (1981b) Compartments in the abdomen of Drosophila and the role of the engrailed locus. Dev Biol 86:363-372.

Kruger R, Kuhn W, Muller T, Woitalla D, Graeber M, Kosel S, Przuntek H, Epplen JT, Schols L, Riess O (1998) Ala30Pro mutation in the gene encoding alpha-synuclein in Parkinson's disease. Nat Genet 18:106-108.

Lieb K, Andersen C, Lazarov N, Zienecker R, Urban I, Reisert I, Pilgrim C (1996) Pre- and postnatal development of dopaminergic neuron numbers in the male and female mouse midbrain. Dev Brain Res 84:37-43

Lin LF, Doherty DH, Lile JD, Bektesh S, Collins F (1993) GDNF: a glial cell line-derived neurotrophic factor for midbrain dopaminergic neurons. Science 260:1130-1132.

Logan C, Willard HF, Rommens JM, Joyner AL (1989) Chromosomal localization of the human homeo box-containing genes, EN1 and EN2. Genomics 4:206-209.

Logan C, Wizenmann A, Drescher U, Monschau B, Bonhoeffer F, Lumsden A (1996) Rostral optic tectum acquires caudal characteristics following ectopic engrailed expression. Curr Biol 6:1006-1014

Lundell MJ, Chu-LaGraff Q, Doe CQ, Hirsh J (1996) The engrailed and huckebein genes are essential for development of serotonin neurons in the Drosophila CNS. Mol Cell Neurosci 7:46-61.

Maroteaux L, Scheller RH (1991) The rat brain synucleins; family of proteins transiently associated with neuronal membrane. Brain Res Mol Brain Res 11:335-343.
Maroteaux L, Campanelli JT, Scheller RH (1988) Synuclein: a neuronspecific protein localized to the nucleus and presynaptic nerve terminal. J Neurosci 8:2804-2815.

Masliah E, Rockenstein E, Veinbergs I, Mallory M, Hashimoto M, Takeda A, Sagara Y, Sisk A, Mucke L (2000) Dopaminergic loss and inclusion body formation in alpha-synuclein mice: implications for neurodegenerative disorders. Science 287:1265-1269.

McMahon AP, Joyner AL, Bradley A, McMahon JA (1992) The midbrain-hindbrain phenotype of Wnt-1-/Wnt-1- mice results from stepwise deletion of engrailed-expressing cells by 9.5 days postcoitum. Cell 69:581-595.

Millen KJ, Wurst W, Herrup K, Joyner AL (1994) Abnormal embryonic cerebellar development and patterning of postnatal foliation in two mouse Engrailed-2 mutants. Development 120:695-706.

Mombaerts P, Wang F, Dulac C, Chao SK, Nemes A, Mendelsohn M, Edmondson J, Axel R (1996) Visualizing an olfactory sensory map. Cell 87:675-686.

Moore MW, Klein RD, Farinas I, Sauer H, Armanini M, Phillips H, Reichardt LF, Ryan AM, Carver-Moore K, Rosenthal A (1996) Renal and neuronal abnormalities in mice lacking GDNF. Nature 382:76-79.

Nosrat CA, Tomac A, Hoffer BJ, Olson L (1997) Cellular and developmental patterns of expression of Ret and glial cell line-derived neurotrophic factor receptor alpha mRNAs. Exp Brain Res 115:410-422.

Paxinos G, Ashwell KWS, Törk I (1994). Atlas of the developing rat nervous system, Ed 2. London: Academic.

Pfaff SL, Mendelsohn M, Stewart CL, Edlund T, Jessell TM (1996) Requirement for LIM homeobox gene Isl1 in motor neuron generation reveals a motor neuron-dependent step in interneuron differentiation. Cell 84:309-320.

Polymeropoulos MH, Higgins JJ, Golbe LI, Johnson WG, Ide SE, Di Iorio G, Sanges G, Stenroos ES, Pho LT, Schaffer AA, Lazzarini AM, Nussbaum RL, Duvoisin RC (1996) Mapping of a gene for Parkinson's disease to chromosome 4q21-q23. Science 274:1197-1199.

Polymeropoulos MH, Lavedan C, Leroy E, Ide SE, Dehejia A, Dutra A, Pike B, Root H, Rubenstein J, Boyer R, Stenroos ES, Chandrasekharappa S, Athanassiadou A, Papapetropoulos T, Johnson WG, Lazzarini AM, Duvoisin RC, Di Iorio G, Golbe LI, Nussbaum RL (1997) Mutation in the alpha-synuclein gene identified in families with Parkinson's disease. Science 276:2045-2047.

Poole SJ, Kornberg TB (1988) Modifying expression of the engrailed gene of Drosophila melanogaster. Development [Suppl] 104:85-93.

Sakurada K, Ohshima-Sakurada M, Palmer TD, Gage FH (1999) Nurr1, an orphan nuclear receptor, is a transcriptional activator of endogenous tyrosine hydroxylase in neural progenitor cells derived from the adult brain. Development 126:4017-4026.

Saueressig H, Burrill J, Goulding M (1999) Engrailed-1 and netrin-1 regulate axon pathfinding by association interneurons that project to motor neurons. Development 126:4201-4212.

Schuchardt A, D'Agati V, Larsson-Blomberg L, Costantini F, Pachnis V (1994) Defects in the kidney and enteric nervous system of mice lacking the tyrosine kinase receptor Ret. Nature 367:380-383.

Sechrist J, Bronner-Fraser M (1991) Birth and differentiation of reticular neurons in the chick hindbrain: ontogeny of the first neuronal population. Neuron 7:947-963.

Simon H, Guthrie S, Lumsden A (1994) Regulation of SC1/DMGRASP during the migration of motor neurons in the chick embryo brain stem. J Neurobiol 25:1129-1143.

Simon H, Hornbruch A, Lumsden A (1995) Independent assignment of antero-posterior and dorso-ventral positional values in the developing chick hindbrain. Curr Biol 5:205-214.

Tanabe Y, Jessell TM (1996) Diversity and pattern in the developing spinal cord. Science 274:1115-1123.

Temlett JA (1996) Parkinson's disease: biology and aetiology. Curr Opin Neurol 9:303-307.

Tomac A, Lindqvist E, Lin LF, Ogren SO, Young D, Hoffer BJ, Olson L (1995) Protection and repair of the nigrostriatal dopaminergic system by GDNF in vivo. Nature 373:335-339.

Voorn P, Kalsbeek A, Jorritsma-Byham B, Groenewegen HJ (1988) The pre- and postnatal development of the dopaminergic cell groups in the ventral mesencephalon and the dopaminergic innervation of the striatum of the rat. Neuroscience 25:857-887.

Wurst W, Auerbach AB, Joyner AL (1994) Multiple developmental defects in Engrailed-1 mutant mice: an early mid-hindbrain deletion and patterning defects in forelimbs and sternum. Development 120:2065-2075.

Ye W, Shimamura K, Rubenstein JL, Hynes MA, Rosenthal A (1998) FGF and Shh signals control dopaminergic and serotonergic cell fate in the anterior neural plate. Cell 93:755-766.

Zetterström RH, Solomin L, Jansson L, Hoffer BJ, Olson L, Perlmann T (1997) Dopamine neuron agenesis in Nurr1-deficient mice. Science $276: 248-250$ 